\title{
Abstract zu: Memes als Wertungen von Literatur in den sozialen Medien
}

Erschienen in: Unterstellte Leseschaften

Von: Berit Glanz

Der Beitrag zeigt, dass in den sozialen Medien neue Formen der Wertung von Literatur und neue Kanonisierungspraktiken entstehen und Memes in diesem Prozess eine entscheidende Rolle spielen.

Die Vervielfältigung der Orte, an denen über literarische Texte diskutiert wird, hat sich durch das Internet noch einmal verstärkt. Das weite Feld des Sprechens über Literatur, des Geschmacksabgleiches, der Diskussion über gute und schlechte Formen literarischen Schreibens ist nicht mehr nur auf analoge Räume beschränkt, sondern verschiebt sich zunehmend in den virtuellen Raum. Durch die vielen Möglichkeiten, online Gehör zu finden, auf Resonanz zu stoßen, sich sein eigenes Publikum aufzubauen, gibt es eine deutliche Ausdifferenzierung des Gesprächs über Literatur. Die Literaturkritik dezentralisiert sich, Wertungskriterien von Literatur und die Literaturkritik selbst werden neu diskutiert und verschieben sich.

In den sozialen Medien gibt es einerseits extrem ausführliche Spezialdiskurse einzelner Stilgemeinschaften. Das heißt beispielsweise die Fans von Liebesromanen diskutieren Werke in ihrem Genre, in ihrer jeweiligen Nische sehr ausführlich und kenntnisreich mit eigenen Kanones und Referenzen. Hierbei etablieren sich auch eigene Formen der Wertschätzung von Büchern, beispielsweise das ritualisierte Auspacken von Neuerscheinungen in Videoclips. Zu den Konventionen von Kommunikation in den sozialen Medien gehören Memes entscheidend dazu. Die Sprachwissenschaftlerin Gretchen McCulloch schreibt: „But one thing we know, if we spend more than a minute discussing internet culture, is that it somehow involves a thing called memes."1 Betrachten wir Memes als wichtigen Aspekt von Internetkultur, dann ist naheliegend zu untersuchen, ob und wie Memes das Gespräch über Literatur in den sozialen Medien prägen und vielleicht sogar eigene Formen der Wertung ausbilden.

Der Beitrag widmet sich dieser Frage anhand verschiedener Beispiele. Zeitgenössische Kanondiskussionen werden in den sozialen Medien mittlerweile auch mit Memes geführt. Am Beispiel von Martin Walser-Memes lässt sich beispielsweise zeigen, dass kanonisierte Autoren als Memes in den sozial-medialen literarischen Diskurs zurückkehren. Hierbei entsteht ein interessantes Paradox: Während beispielsweise auf Twitter sehr viel Zeit darauf verwendet wird, sich spöttisch und humorvoll von einem literarischen Kanon abzugrenzen, neue Kriterien zu definieren und sich gegenseitig zu 
versichern, dass man tatsächlich die Freiheit habe, sich von etablierten Wertungskriterien abzuwenden, ist die Kenntnis kanonisierter Autor*innen oftmals dennoch wichtig, um bestimmte Memes überhaupt zu verstehen.

Ein wichtiger Teil des literarischen Diskurses in Foren und sozialen Medien ist auch die spielerische Dekanonisierung in Form von Parodien bekannter Autor*innen und etablierter Schreibweisen. Durch Retweets und Favs werden die in der Parodie implizit geäußerten Wertungen dann bestätigt und beeinflussen den Literaturbegriff der sich konstituierenden Fav-Gemeinschaft.

Ein weiterer Bereich literarischer Wertung und Kanonisierung durch Memes in den sozialen Medien ist die Memifizierung spezifischer literarischer Texte. Memes, die Literatur als Vorlage nehmen, oszillieren immer zwischen Spott und Anerkennung, lassen sich kritisch oder affirmativ lesen. Memifizierung enthält nicht grundsätzlich eine kritische oder positive Wertung, sondern es muss anhand von Einzelfällen betrachtet werden, wie diese Memes entstehen, sich entwickeln und verbreiten, ob sie kritisch oder affirmativ gelesen werden können.

Die Langfassung dieses Beitrages in der Reihe „Unterstellte Leseschaften“ finden Sie hier.

\section{References}

1. Gretchen McCulloch: Because Internet: Understanding the New Rules of Language. New York 2019, S. 238.

SUGGESTED CITATION: Glanz, Berit: Abstract zu: Memes als Wertungen von Literatur in den sozialen Medien, in: KWI-BLOG, [https://blog.kulturwissenschaften.de/abstract-zu-memes-als-wertungen-von-literatur-inden-sozialen-medien/], 03.05.2021

DOI: https://doi.org/10.37189/kwi-blog/20210503-0832 


\section{DuEPublico}

Duisburg-Essen Publications online

\section{DESISEN R G}

offen im Denken

$\mathbf{U b} \mid \begin{aligned} & \text { universitäts } \\ & \text { bibliothek }\end{aligned}$

Dieser Text wird via DuEPublico, dem Dokumenten- und Publikationsserver der Universität Duisburg-Essen, zur Verfügung gestellt. Die hier veröffentlichte Version der E-Publikation kann von einer eventuell ebenfalls veröffentlichten Verlagsversion abweichen.

DOI: $\quad$ 10.37189/kwi-blog/20210503-0832

URN: urn:nbn:de:hbz:464-20210503-164247-9 